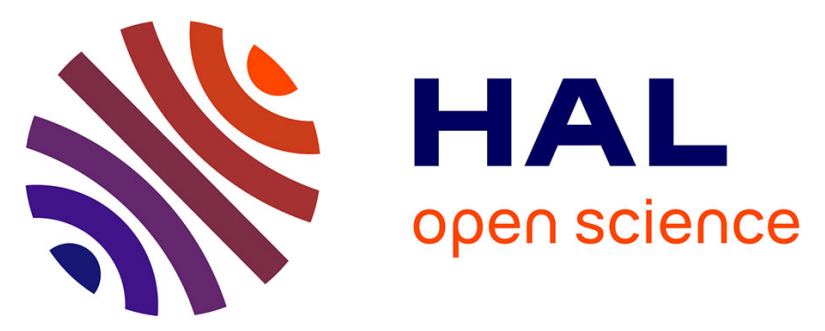

\title{
Feedback linearizing controller coupled to an Unscented Kalman filter for lactic acid regulation
}

\author{
Karen Vanessa Gonzalez, Sihem Tebbani, Didier Dumur, Filipa Lopes, \\ Dominique Pareau, Aurore Thorigné, Sébastien Givry
}

\section{- To cite this version:}

Karen Vanessa Gonzalez, Sihem Tebbani, Didier Dumur, Filipa Lopes, Dominique Pareau, et al.. Feedback linearizing controller coupled to an Unscented Kalman filter for lactic acid regulation. 19th International Conference on System Theory, Control and Computing (ICSTCC), Oct 2015, Cheile Gradistei, Romania. 10.1109/ICSTCC.2015.7321296 hal-01257002

\section{HAL Id: hal-01257002 \\ https://hal-centralesupelec.archives-ouvertes.fr/hal-01257002}

Submitted on 12 Mar 2020

HAL is a multi-disciplinary open access archive for the deposit and dissemination of scientific research documents, whether they are published or not. The documents may come from teaching and research institutions in France or abroad, or from public or private research centers.
L'archive ouverte pluridisciplinaire HAL, est destinée au dépôt et à la diffusion de documents scientifiques de niveau recherche, publiés ou non, émanant des établissements d'enseignement et de recherche français ou étrangers, des laboratoires publics ou privés. 


\title{
Feedback Linearizing Controller Coupled to an Unscented Kalman Filter for Lactic Acid Regulation
}

\author{
Karen Gonzalez, Sihem Tebbani, Didier Dumur \\ Laboratoire des Signaux et Systèmes (L2S, UMR CNRS \\ 8506) CentraleSupélec-CNRS-Université Paris-Sud, \\ Control Departement \\ F 91192 Gif sur Yvette cedex, France \\ karen.gonzalez@centralesupelec.fr, \\ sihem.tebbani@centralesupelec.fr, \\ didier.dumur@centralesupelec.fr
}

\author{
Karen Gonzalez, Filipa Lopes, Dominique Pareau \\ Laboratoire de Génie des Procédés et Matériaux \\ Ecole CentraleSupélec \\ F 92295 Châtenay-Malabry cedex, France \\ filipa.lopes@centralesupelec.fr, \\ dominique.pareau@centralesupelec.fr
}

\author{
Aurore Thorigné, Sebastien Givry \\ Centre de Recherche et d'Innovation Soufflet-Division Biotechnologie-Groupe Soufflet \\ F10400, Nogent sur Seine cedex, France \\ athorigne@soufflet-group.com, sgivry@soufflet-group.com
}

\begin{abstract}
This paper deals with the development of a control law for the regulation of the lactic acid concentration in its biotechnological production process. The studied system consists in a continuous bioreactor using wheat flour as raw material. Three reactions take place at the same time in the bioreactor: the gluten hydrolysis, the maltose saccharification and the glucose fermentation. This paper considers the optimization of the two last reactions. The optimal set point that maximizes the bioprocess productivity is first determined. Then, a state feedback linearizing controller is developed to maintain the lactic acid concentration at this optimal set point. Nevertheless, knowledge of all states variables is required for this kind of control law. Since the sole online measurement is the lactic acid concentration, robust and efficient estimators of the nonmeasured states have to be developed. In this paper, an Unscented Kalman filter (UKF) is proposed to estimate the biomass and substrates concentrations in the bioreactor and is then coupled with the feedback linearizing control law. Numerical simulations have been carried out to assess the control law performance.
\end{abstract}

Keywords-Lactic acid regulation; wheat flour; state feedback linearizing control; Unscented Kalman Filter

\section{INTRODUCTION}

The expanding Lactic acid use as the first monomer for the PLA (Poly Lactic Acid) production demands more efficient and reliable processes to optimize its production. As lactic acid is a cheap product, the use of non-expensive raw materials is desired. Wheat flour has been reported as suitable for the lactic acid production in large-scale [1]. The major factors affecting lactic acid production are the nutrient limiting conditions and the inhibitory effect caused by lactic acid accumulation in the bioreactor. To face the first problem, the fermentation phase proposed in this work comprises a Simultaneous Saccharification, Proteins Hydrolysis and
Fermentation (SSPHF) in order to guarantee the availability of the substrates during the process. The starch was pretreated and transformed into glucose and maltose (this step is out of the scope of this paper). Three reactions take place at the same time in the SSPHF step: the gluten and glucose hydrolysis, the maltose saccharification and the production of lactic acid by bacteria. The inhibitory effect by the product can be avoided by operating the bioreactor in continuous mode.

Kinetic models are important tools for optimizing biotechnological processes and to predict bioreactor's performance. In this work, we consider the SSPHF bioreactor modelling taking into account the maltose saccharification and the glucose fermentation. The proteins hydrolysis kinetics was neglected as it was not the limiting substrate in the bioreactor. One way to optimize the bioreactor performance is by means of control strategies which allow regulating the key components in the bioreactor. Nevertheless, as the model used for this biotechnological process exhibits strong nonlinearities, the development of a control strategy for the continuous bioreactor is challenging. Some authors have proposed control strategies for fermentation processes and obtained satisfactory results [2][3][4][5]. However, the processes studied concern fed-batch fermentation or use of simpler culture media than the one considered in this work. The challenge of our work is to deal with the simultaneous production and consumption of glucose in the development of the control law.

The control objective is to regulate the lactic acid concentration at an optimal value using the feed flow rate as the control variable. A state feedback linearizing controller is developed to track the optimal set point. However, online knowledge of all state variables is required. The sole variable measured online in the bioreactor is the lactic acid concentration, thus estimation of the non-measured variables is necessary. The main approaches for bioprocess estimation 
are based on asymptotic estimator [6], extended Kalman filter [7], and interval observers [8]. The most widely used estimator in the literature is the extended Kalman filtering (EKF). However, the linearization approach in this algorithm hinders its implementation, particularly when the model has strong nonlinearities. Indeed, the linearization of the process dynamics can be a complex task. An alternative is to use the Unscented Kalman Filtering (UKF) proposed by Julier and Uhlmann [9]. This observer uses a similar approach as the EKF, avoiding the linearization procedure and leading to a better robustness and speed of convergence [10]. In this paper, an UKF is proposed for states estimation and coupled to the feedback linearizing controller. In a previous work [11], the linearizing control strategy was tested for the considered system and its performance highlighted in simulation. The state variables were assumed to be measured online. This paper will complete the previous study by developing an estimation strategy to reconstruct the non-measured state variables. The performance of the control strategy when coupled with an UKF will be then studied to assess the efficiency of the developed control law. In addition, in this paper, the maltose saccharification is considered on the contrary of [11].

This paper is structured as follows: In section 2, the bioreactor description and modelling is presented. In Section 3 , the linearizing state feedback strategy to regulate the lactic acid concentration is developed. The UKF development for the biomass and glucose concentrations estimation is presented in Section 4. Numerical simulations and robustness analysis are presented in Section 5. Conclusions are given in Section 6.

\section{BIOREACTOR MODELLING}

\section{A. Process Description}

The lactic acid production from starch is a complex process. Indeed, the degradation of starch must be performed in order to provide the glucose (carbon substrate) to bacteria. In fact, bacteria cannot use the starch directly as its carbon source. A pre-treatment of starch by gelatinisation and liquefaction is then necessary. This step yields mainly to maltose which must be hydrolysed to glucose enzymatically. In the liquefaction step, starch is degraded to maltose by the alpha-amylase enzyme. Later, maltose is converted to glucose by the amyloglucosidase enzyme (saccharification). Finally, bacteria transform the glucose into lactic acid by fermentation [12]. This work considers bacteria Lactobacillus coryniformis subsp. Torquens, a homofermentative strain. In this work, the final part of the saccharification step is combined with the fermentation. It means that maltose is partially hydrolysed in the saccharification step and then the remaining maltose is hydrolysed simultaneously with the fermentation (SSPHF). The bioreactor used for the SSPHF is studied in this paper. It will be operated in continuous mode. A schematic diagram of the key variables is depicted in Fig. $1 . M_{0}$ and $S_{0}$ are maltose and glucose concentrations feeding the bioreactor, respectively. The key variables in the bioreactor are the maltose concentration $(M)$, the glucose concentration $(S)$, the biomass concentration $(X)$ and the lactic acid concentration $(P)$. All concentrations are in $\mathrm{g} / \mathrm{L}$. The temperature and $\mathrm{pH}$ are regulated to 5.7 and $30^{\circ} \mathrm{C}$ respectively.

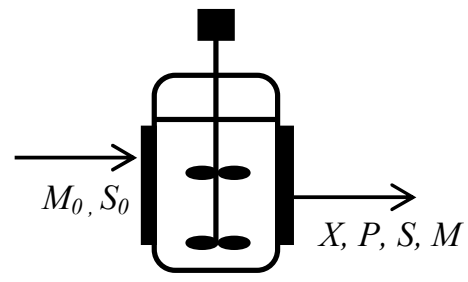

Fig. 1. Representation of the SSPHF bioreactor

\section{B. Model development}

Glucose in the bioreactor is simultaneously produced (by maltose saccharification) and consumed (by bacteria). The reaction scheme can be modeled in general as $k_{1} S \stackrel{r}{\longrightarrow} X+k_{2} P$, where $k_{i}, i=1,2$ are the stoichiometric coefficients and $r$ the reaction rate. The mathematical model to describe the concentrations evolution must take into account the maltose contribution in glucose dynamics. A model is considered hereafter including four dynamic equations: biomass growth, substrate consumption, product formation and maltose degradation. Dynamics of these variables are based on mass balance equations for a continuous perfectly stirred bioreactor. The system dynamics are then given by:

$$
\begin{gathered}
\dot{X}=\mu X-D X \\
\dot{S}=-\frac{1}{Y_{X}} \mu X-D\left(S-S_{0}\right)+k_{M} M \\
\dot{P}=\frac{Y_{P}}{Y_{X}} \mu X-D P \\
\dot{M}=-k_{M} M-D\left(M-M_{0}\right)
\end{gathered}
$$

where $\mu$ is the specific growth rate (in $\mathrm{h}^{-1}$ ), $D$ is the dilution rate $\left(\mathrm{h}^{-1}\right)$ defined as the ratio of the feed flow rate over the effective reactor volume. $Y_{P}$ and $Y_{X}$ are product and biomass yields with respect to glucose respectively (in $\mathrm{g} / \mathrm{g}$ ), $k_{M}$ is the maltose degradation constant (in $\mathrm{h}^{-1}$ ). In this work, the substrate limitation and product inhibition effects on cells growth are considered. The cell specific growth rate is then described by [13]:

$$
\mu=\mu_{\max } \frac{S}{k_{S}+S}\left(1-\frac{P}{P_{\max }}\right)^{n}
$$

where $\mu_{\text {max }}$ is the maximal specific growth rate (in $\left.\mathrm{h}^{-1}\right), k_{S}$ is the half saturation constant $(\mathrm{g} / \mathrm{L}), n$ the lactic acid inhibitor power and $P_{\max }$ the maximal lactic acid concentration above which bacteria do not grow (in $\mathrm{g} / \mathrm{L}$ ). The term in (2) involving the substrate concentration represents the growth limitation by the substrate according to the Monod model. The second term models the growth inhibition by the product (i.e. for high product concentration, the growth rate decreases).

As the lactic acid production in the bioreactor decreases the $\mathrm{pH}$ value, the latter is regulated at 5.7 during the SSPHF by the addition of a base solution of $\mathrm{NaOH}$. This value of $\mathrm{pH}$ 
was determined by experimental assays so that the bacteria growth is maximized. The added quantity of $\mathrm{NaOH}$ allows to determine online the lactic acid concentration (each 5 minutes). These experimental assays show that the lactic acid concentration is well estimated by this approach (good accuracy and very low measurement noise).

To summarize, the studied system is then made of 4 state variables, with a temporal evolution described by (1), only the product concentration being available online.

\section{CONTROL STRATEGY}

\section{A. Optimal set point}

The proposed control strategy consists in modifying the dilution rate in real time so that the lactic acid concentration is maintained at a specified optimal set point. At steady state conditions, the dilution rate equals the growth rate. To determine the concentrations of the variables at the steady state, the specific growth rate given by (2) was approximated using the following expression:

$$
\bar{D}=\mu \approx \mu_{\max }\left(1-\frac{\bar{P}}{P_{\max }}\right)^{n}
$$

where $\bar{D}$ and $\bar{P}$ are the steady state dilution rate and lactic acid concentration, respectively. Indeed, substrate is not limiting in the considered operating conditions, and thus its effect on cell growth can be neglected. This simplification is also valid in transient conditions. The steady state of the state variables can be determined by cancelling dynamics in (1) and from (3). The following equations that describe the state variables at steady state as a function of the dilution rate are derived:

$$
\begin{gathered}
\bar{P}=\left(-\frac{\bar{D}^{1 / n}}{\mu_{\max }^{1 / n}}+1\right) P_{\max } \\
\bar{X}=\frac{Y_{X}}{Y_{P}}\left(-\frac{\bar{D}^{1 / n}}{\mu_{\text {max }}^{1 / n}}+1\right) P_{\max } \\
\bar{M}=\frac{\bar{D} M_{0}}{\left(\bar{D}+K_{M}\right)} \\
\bar{S}=\frac{K_{M} M_{0}}{\left(\bar{D}+K_{M}\right)}+S_{0}-\frac{1}{Y_{P}}\left(-\frac{\bar{D}^{1 / n}}{\mu_{\max }^{1 / n}}+1\right) P_{\max }
\end{gathered}
$$

where $\bar{X}, \bar{M}$ and $\bar{S}$ are the biomass, the maltose and the glucose concentrations at steady state, respectively.

The optimal set point is calculated to maximize lactic acid productivity, defined as the product of the steady state dilution rate by the steady state lactic acid concentration $(\bar{D} * \bar{P})$. The determination of this optimal set point can be formulated as the solution of a constrained optimization problem:

$$
\begin{aligned}
& \bar{P}^{*}=\arg \max \bar{D} \cdot \bar{P} \\
& \text { s.t. } \quad(3) \\
& 0 \leq \bar{D} \leq D_{\max }
\end{aligned}
$$

where $D_{\max }$ is the maximal dilution rate allowed by the experimental setup. Figure 2 gives the evolution of the productivity $\bar{D} * \bar{P}$ for different values of steady state product concentrations. This function is quasi-concave and has only one maximum, simplifying the optimization procedure. The numerical optimization of problem (8) was presented in [11] in the general case. Hereafter, the bioreactor will be maintained at this optimal set point by regulating the lactic acid concentration at its optimal value (here it equals $20 \mathrm{~g} / \mathrm{L}$ ).
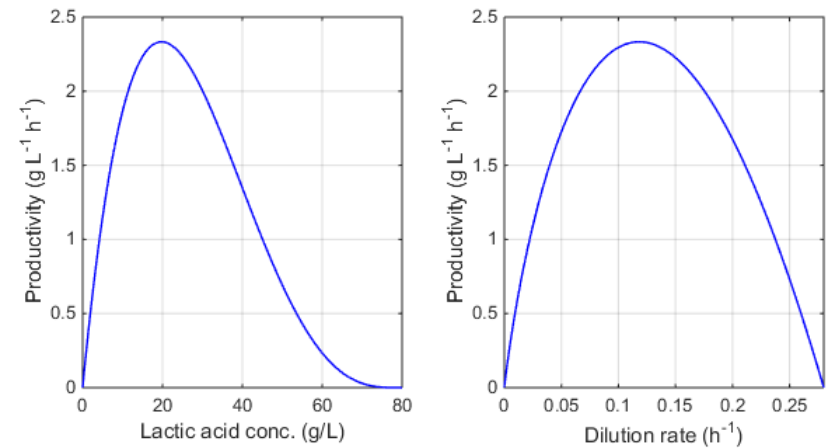

Fig. 2. Evolution of lactic acid productivity with steady state lactic acid concentration.

\section{B. Control Model}

The system (1) can be rewritten in the state-space formalism as follows:

$$
\begin{aligned}
& \dot{x}(t)=f(x)+g(x) u \\
& y(t)=h(x)
\end{aligned}
$$

with $x=(X, S, P, M)^{T}$ the state vector, $u=D$ the control input and $y=P$ the output. As the system (9) is a controlaffine one, a state feedback linearizing controller can be used [14]. A proportional controller is also implemented in the outer loop. For the implementation of the linearizing controller, all state variables must be available (either measured or estimated online).

In order to design the linearizing controller, the relative degree of system (9) was determined [14]. It is denoted by $r$ (with $r \leq n=\operatorname{dim}(x)$ ). The input $u$ is related to the linearized system input $\hat{u}$ by:

$$
u=\frac{\hat{u}-L_{f}^{r} h(x)}{L_{g} L_{f}^{r-1} h(x)}
$$

where $L$ is the Lie derivative with $L_{f} h(x)=\frac{\partial h}{\partial x} f(x)$ and $L_{g} h(x)=\frac{\partial h}{\partial x} g(x)$.

When $r<n$, the system is partially linearizable and is equivalent to $r$ integrators, using relation (10). In the case of the studied system given by (1), the relative degree is found to be $r=1$. Thus, only the product concentration dynamics can be linearized. The control input is then derived from (10) and (1c), leading to: 


$$
D=-\frac{1}{P}\left[\hat{D}-Y_{P} \frac{\mu}{Y_{X}} X\right]
$$

The control signal $\hat{D}$ will be delivered by the outer loop by means of a Proportional controller:

$$
\hat{D}=G\left(P_{\text {ref }}-P\right)
$$

where $P_{r e f}$ is the reference product concentration (the solution of (8)) and $G$ is the controller gain tuned to provide a desired closed-loop time response. Indeed, the linearized system by the feedback control law is equivalent to an integrator, leading to a closed-loop system with transfer function that equals $1 /(1+\mathrm{s} / \mathrm{G})$, where $\mathrm{s}$ is the Laplace transform.

\section{State variables estimation}

Since only the lactic acid concentration is measured online, it is necessary to develop an observer to reconstruct nonmeasured concentrations from this measurement. First, the observability of the state variables from the lactic acid measurement must be checked. The observability analysis for the system (1) shows that state variables $x$ are observable from measurements of output variable $P$. In this work, an Unscented Kalman Filter (UKF) is implemented for the estimation of state variables. The UKF algorithm is presented in Appendix. The estimated concentrations are then used by the state feedback linearizing controller (see Fig. 3).

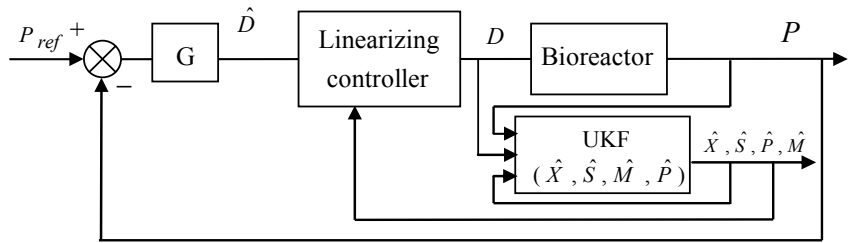

Fig. 3. Control strategy architecture with UKF.

The studied system is characterized by nonlinear differential equations. The continuous system in equations 1 can be then discretized by the Euler method considering that the dynamics evolution of the system is slow enough (in comparison to the chosen sampling time). The discretized representation of the system with a sampling period $T_{s}=5 \mathrm{~min}$ is governed by the following equations:

$$
\left\{\begin{array}{l}
X_{k+1}=X_{k}+T_{s}\left(\mu_{k} X_{k}-D_{k} X_{k}\right) \\
S_{k+1}=S_{k}\left(-D_{k} T_{s}+1\right)+T_{S}\left(-\frac{1}{Y_{X S}} \mu_{k} X_{k}+D S_{0}+k_{M} M\right) \\
M_{k+1}=M_{k}\left(-D_{k} T_{s}-k_{M} T_{s}+1\right)+T_{S}\left(D_{k} M_{0}\right) \\
S_{k+1}=P_{k}\left(-D_{k} T_{s}+1\right)+T_{S}\left(\frac{Y_{P S}}{Y_{X S}} \mu_{k} X_{k}\right) \\
\mu_{k}=\mu_{\max } \frac{S_{k}}{k_{S}+S_{k}}\left(1-\frac{P_{k}}{P_{\max }}\right)^{n}
\end{array}\right.
$$

The UKF principle can then be applied to the system by adding fictitious state and measurements noises to the equations system (13) as in (15) (see Appendix).

\section{NUMERICAL RESUlTS}

In this section, the proposed control strategy is tested and validated in simulation. The model parameters were determined from experimental data of a SSPHF performed in continuous mode, at $\mathrm{pH}=5.7$ and temperature at $30^{\circ} \mathrm{C}$, with the identification strategy proposed in [15]. Parameter values are given in Table I, and simulation conditions in Table II.

TABLE I. MODEL PARAMETERS VALUES

\begin{tabular}{|l|c|}
\hline Parameter & $\begin{array}{c}\text { Identified } \\
\text { Value }\end{array}$ \\
\hline$\mu_{\max }\left(\mathrm{h}^{-1}\right)$ & 0.28 \\
\hline$Y_{X}(\mathrm{~g} / \mathrm{g})$ & 0.05 \\
\hline$Y_{P}(\mathrm{~g} / \mathrm{g})$ & 0.82 \\
\hline$k_{M}\left(\mathrm{~h}^{-1}\right)$ & 0.035 \\
\hline$k_{S}(\mathrm{~g} / \mathrm{L})$ & 0.50 \\
\hline$P_{\max }(\mathrm{g} / \mathrm{L})$ & 79.29 \\
\hline$n$ & 3 \\
\hline
\end{tabular}

TABLE II. SimULATION CONDITIONS

\begin{tabular}{|l|l|}
\hline Variable & Value \\
\hline$P(0)(\mathrm{g} / \mathrm{L})$ & 0 \\
\hline$X(0)(\mathrm{g} / \mathrm{L})$ & 0.2 \\
\hline$S(0)(\mathrm{g} / \mathrm{L})$ & 120 \\
\hline$M(0)(\mathrm{g} / \mathrm{L})$ & 50 \\
\hline$\hat{P}(0)(\mathrm{g} / \mathrm{L})$ & 0.1 \\
\hline$\hat{S}(0)(\mathrm{g} / \mathrm{L})$ & 110 \\
\hline$\hat{X}(0)(\mathrm{g} / \mathrm{L})$ & 0.5 \\
\hline$\hat{M}(0)(\mathrm{g} / \mathrm{L})$ & 55 \\
\hline$D_{\max }\left(\mathrm{h}^{-1}\right)$ & 0.4 \\
\hline$P_{r e f}(\mathrm{~g} / \mathrm{L})$ & 20 \\
\hline$S_{0}(\mathrm{~g} / \mathrm{L})$ & 120 \\
\hline$M_{0}(\mathrm{~g} / \mathrm{L})$ & 50 \\
\hline$G$ & 6 \\
\hline
\end{tabular}

Two control laws were tested and compared. The first one considers the classical feedback linearizing controller given by (11-12) (referred to as Ideal), where all state variables are assumed to be measured online. In the second one, the feedback linearizing controller is coupled to the UKF. The aim is to regulate the lactic acid concentration to its optimal value $P_{r e f}=20 \mathrm{~g} / \mathrm{L}$. Initial values considered for the real system differ from those used in the UKF in order to highlight estimator robusteness with respect to initialization errors. Simulations consider a random $15 \%$ non-correlated parameter mistmatch in the real system (i.e. parameters of the real process differ from those used in the estimator and in the control law). This will help to test robusteness of the estimation and control law with respect to model mismatch. In addition, the measured product concentration is assumed to be corrupted with a gaussian white noise of zero mean and 0.1 standard deviation. The UKF tuning parameters are chosen as follows: $Q=\operatorname{diag}([0.01 ; 10 ; 1]), \quad R=0.01, \quad \mathrm{P}_{0}=\operatorname{diag}([0.1 ; 1 ; 0.1]), \quad \beta=2$, 
$\alpha=0.5$ and $k_{0}=0$. These values were determined either from measurement characteristics or from a trial/error technique.

Obtained results when applying the two control laws are presented in Figs. 5 and 6 . When the fermentation starts, the bioreactor operates in batch mode in order to increase the lactic acid concentration (Fig. 4). Once this concentration is close to the desired set point, a dilution is applied in the bioreactor in order to dilute the product concentration and maintain its value at the desired optimal point. The set point is reached after about $10 \mathrm{~h}$ with good transient behavior. It can be noticed that the noise on the measurement leads to some fluctuations on the control input. This phenomena could be reduced by filtering the output.

The comparison of the "ideal" trajectory (obtained with the ideal control) with the one obtained with the controller coupled with UKF, shows that the two trajectories are very close. However, including the UKF leads to a slight steady state error (see zoom in Fig. 4). This offset can be corrected by the implementation of an integral controller in the outer-loop of the control strategy. Generally, an anti-windup device is also necessary because of control input saturation [11]. Nevertheless, the proposed control law that includes the UKF leads to good performances in terms of accuracy and stability. The state variables predicted by the model (with parameter mistmach) and their estimated values given by the UKF are compared in Fig. 5. The UKF estimation of the biomass, glucose and maltose concentrations is satisfactory. Nevertheless a steady state error is present due to the initialisation error and the model mismatch. It can be noticed that all the inlet quantity of glucose is not consumed. Indeed, this high value of glucose concentration in the feeding medium was determined to allow connecting, in the future, several SSPHF bioreactors in series.

In conclusion, the state feedback linearizing controller coupled to the UKF shows good performances to regulate the lactic acid concentration.
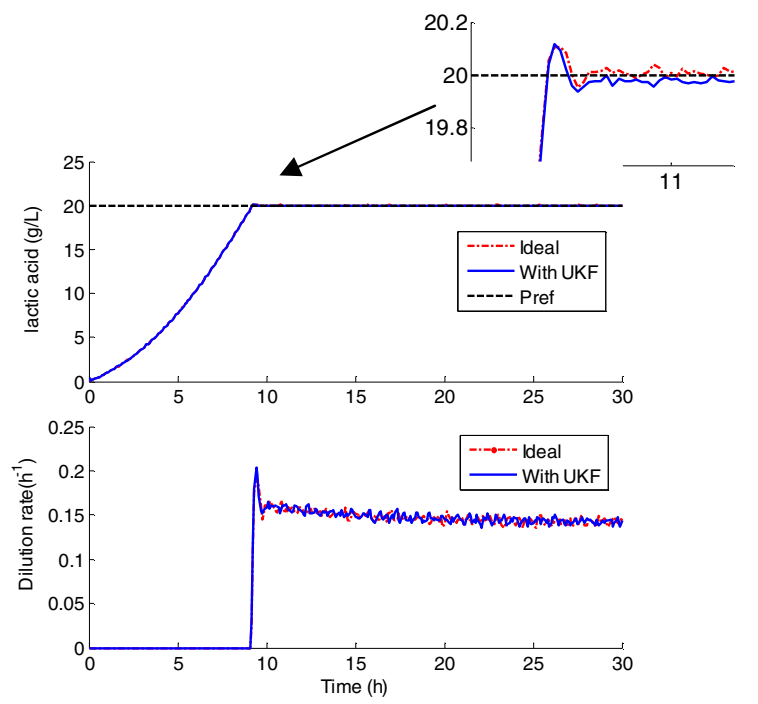

Fig. 4. Control law performenaces for reference tracking. Evolution of product concentration and dilution rate with time. Model and intial state values mistmatch between the real system and the considered model.
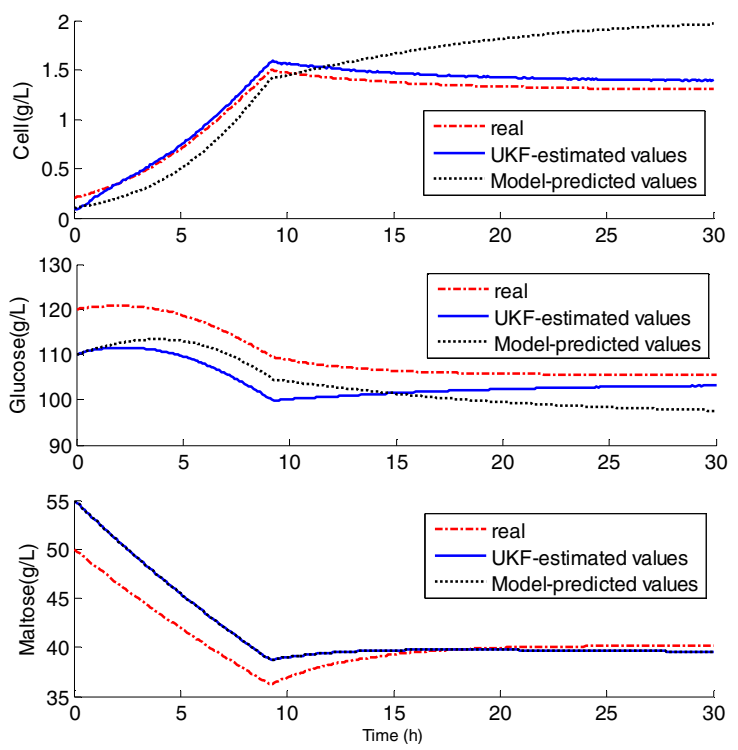

Fig. 5. State variables evolution with time. Model and initial values mistmatch between real system and the considered model.

\section{CONCLUSIONS}

This paper considers the optimization of a simultaneous saccharification and fermentation process for lactic acid production in continuous mode. First, the optimal steady state is determined so that the lactic acid productivity is maximized. Then, a control strategy is proposed, based on the feedback linearizing control law combined with an upper level linear proportional controller. This control strategy regulates the lactic acid concentration to the determined optimal value. In order to estimate the glucose and biomass concentrations, an Unscented Kalman Filter was developed and coupled to the control law. The proposed controller combined to the UKF shows good performances in terms of accuracy and robustness. Further study will consider the comparison of the proposed control strategy with other kinds of controllers (with estimation of production rate for a kinetic free controller).

\section{REFERENCES}

[1] K. Hetényi, A. Németh, and B. Sevella, First steps in the development of a wheat flour based lactic acid fermentation technology. Culture medium optimization. Chemical and Biochemical Engineering Quarterly, vol. 24, pp. 195-201., 2010

[2] J. F. Van Impe and G. Bastin, Optimal adaptive control of fed-batch fermentation processes. Control Eng. Practice, vol. 7, pp. 939-954, 1995.

[3] C. Ben Youssef, V. Guillou and A. Olmos-Dichara, Modelling and adaptive control strategy in a lactic fermentation process. Control Engineering Practice, vol. 11, pp.1297-1307, 2000.

[4] S. Ochoa, V. Lyubenova, J. U. Repke, M. Ignatova and G. Wozny, Adaptive control of the simultaneous saccharification-fermentation process from starch to ethanol. Computer Aided Chemical Engineering, vol. 25, pp. 257-269, 2008.

[5] E. Petre, D. Selişteanu and D. Şendrescu, Neural networks based adaptive control of a fermentation bioprocess for lactic acid production, In Proceedings of 3rd International Conference on Intelligent Decision Technologies, SIST 10, pp 201-212.

[6] G. Bastin and D. Dochain, Online estimation and adaptive control of bioreactors, Process Measurement and Control, vol. 1. Elsevier, Amsterdam, 1990. 
[7] S. Tebbani, F. Lopes and G. Becerra Celis, Nonlinear control of continuous cultures of Porphyridium pupureum in a photobioreactor. Chemical Engineering Science, vol. 123, pp. 207-219, 2015.

[8] A. Rapaport and D. Dochain, Interval observers for biochemical processes with uncertain kinetics and inputs. Mathematical Biosciences, vol. 193 no. 2 , pp. 235-253,2005.

[9] S. J. Julier and J.K. Uhlmann, A new extension of the Kalman filter to nonlinear systems, In Proceedings of International Symp. Aerospace/Defense sensing, Simul. And Controls, 182-193,1997.

[10] R. Kandepu, B. Foss and L. Imsland, Applying the unscented Kalman filter for nonlinear state estimation. Journal of Process Control, vol. 18, 753-768, 2008.

[11] K. Gonzalez, S. Tebbani, D. Dumur, D. Pareau, F. Lopes, S. Givry and F. Entzmann, Control strategy for continuous lactic acid production from wheat flour. In Proceedings of 2nd International Conference on Control, Decision and Information Technologies, Metz, France, 2014.

[12] R. Anuradha, A. K. Suresh and K. V. Venkatesh, Simultaneous saccharification and fermentation of starch to lactic acid. Process Biochemistry, vol. 35, pp.367-375, 1999.

[13] S. Kumar Dutta, A. Mukherjee and P. Chakraborty, Effecto of product inhibition on lactic acid fermentation: Simulation and modelling. Applied Microbiology and Bioetchnology, vol. 45, pp.410-413.

[14] A. Isidori, Nonlinear Control Systems. Springer-Verlag, Berlin. 1989.

[15] K. Gonzalez, S. Tebbani, D. Dumur, D. Pareau, F. Lopes, S. Givry and F. Entzmann, Modeling and parameter identification of the batch lactic acid production process from wheat flour. In Proceedings of $17 \mathrm{th}$ International Conference on System Theory, Control and Computing ICSTCC 2013, Romania, 2013.

\section{APPENDIX :UnSCENTED KALMAN FILTER ALgORITHM}

An estimator is needed for control purposes. A widely estimation technique used in biotechnological process is the EKF. In this approach, the state distribution is propagated analytically through the first-order linearization of the nonlinear system. As the system (1) has strong nonlinearities and uncertainties, the derivative-free UKF method is preferred. In this case, the state distribution is represented by means of a minimal set of carefully chosen sampling points, the sigma points. Each of these points is propagated through the nonlinearities, the mean and the covariance being taken out from these transformed points (Julier and Uhlmann, 1997). The UKF algorithm is detailed hereafter.

Let consider the discrete-time nonlinear model:

$$
\begin{aligned}
& x_{k+1}=F\left(x_{k}, u_{k}\right)+v_{k} \\
& y_{k}=H\left(x_{k}\right)+w_{k}
\end{aligned}
$$

where $x$ is the state vector $(\operatorname{dim}(x)=n), u$ the input, $F$ and $H$ are nonlinear functions, $v_{k}$ is the process noise and $w_{k}$ is the measurement noise.

The UKF algorithm is given by:

Initialization $(k=0)$ :

$$
\hat{x}_{0}=E\left[x_{0}\right], \mathbf{P}_{0}=E\left[\left(x_{0}-\hat{x}_{0}\right)\left(x_{0}-\hat{x}_{0}\right)^{T}\right]
$$

For $k=1, \ldots, \infty$ :

Step 1. Sigma points selection :

$$
\begin{gathered}
\left(\xi_{k-1}\right)_{0}=\hat{x}_{k-1} \\
\left(\xi_{k-1}\right)_{i}=\hat{x}_{k-1}+\gamma \cdot\left(\sqrt{\mathbf{P}_{k-1}}\right)_{i}, i=1, \ldots, n, \\
\left(\xi_{k-1}\right)_{i}=\hat{x}_{k-1}-\gamma \cdot\left(\sqrt{\mathbf{P}_{k-1}}\right)_{i-n}, i=n+1, \ldots, 2 n,
\end{gathered}
$$

where $\left(\sqrt{\mathbf{P}_{k-1}}\right)_{i}$ is the $\mathrm{i}$-th column of the square root of the covariance matrix of the previous time step (calculated by Cholesky factorization). The $\gamma$ parameter is the scaling factor used to move the position of the sigma points around the mean value and is given by $\gamma=\sqrt{n+\lambda}$, with $\lambda=\alpha^{2}\left(n+k_{0}\right)-n$ and $\left(\alpha, k_{0}\right)$ are parameters to choose. Usually $k_{0}$ is set to zero and $\alpha$ is small $\left(10^{-4} \leq \alpha \leq 1\right)$.

Step 2. Prediction. In this step the sigma points $\left(\xi_{k-1}\right)$ are propagated through the nonlinear dynamics and the state prediction is calculated:

$$
\begin{aligned}
& \boldsymbol{\xi}_{k \mid k-1}=F\left[\boldsymbol{\xi}_{k-1}, u_{k-1}\right] \\
& \hat{x}_{k}^{-}=\sum_{i=0}^{2 n} W_{i}^{(m)} \xi_{i, k \mid k-1}
\end{aligned}
$$

The predicted covariance is computed using the following expression:

$$
\mathbf{P}_{k}^{-}=\sum_{i=0}^{2 n} W_{i}^{(c)}\left[\xi_{i, k \mid k-1}-\hat{x}_{k}^{-}\right]\left[\xi_{i, k \mid k-1}-\hat{x}_{k}^{-}\right]^{T}+Q
$$

where $Q$ is the process noise covariance matrix.

Step 3. Updating. Using the predicted sigma points (18) and covariance (20), a new set of sigma points is computed and projected through the observation model. The predicted measurements are described by:

$$
\begin{gathered}
Y_{k \mid k-1}=H\left[\xi_{k-1}\right] \\
\hat{y}_{k}^{-}=\sum_{i=0}^{2 n} W_{i}^{(m)} Y_{i, k \mid k-1}
\end{gathered}
$$

The covariance of the innovation and the cross-covariance matrix are then given by:

$$
\begin{aligned}
\mathbf{P}_{\tilde{y}_{k} \tilde{y}_{k}} & =\sum_{i=0}^{2 n} W_{i}^{(c)}\left[Y_{i, k \mid k-1}-\hat{y}_{k}^{-}\right]\left[Y_{i, k \mid k-1}-\hat{y}_{k}^{-}\right]^{T}+R \\
\mathbf{P}_{y_{k} x_{k}} & =\sum_{i=0}^{2 n} W_{i}^{(c)}\left[\xi_{i, k \mid k-1}-\hat{x}_{k}^{-}\right]\left[Y_{i, k \mid k-1}-\hat{y}_{k}^{-}\right]^{T}
\end{aligned}
$$

where $R$ is the measurement noise covariance.

The estimations are updated using the classical Kalman filter equations:

$$
\begin{gathered}
K_{k}=\mathbf{P}_{y_{k} x_{k}} \mathbf{P}_{y_{k} y_{k}}^{-1} \\
\hat{x}_{k}=\hat{x}_{k}^{-}+K_{k}\left(y_{k}-\hat{y}_{k}^{-}\right) \\
\mathbf{P}_{k}=\mathbf{P}_{k}^{-}-K_{k} \mathbf{P}_{\tilde{y}_{k} \tilde{y}_{k}} K_{k}^{T}
\end{gathered}
$$

Step 4. Repeat Steps 1 to 3 for the next sample.

The weighting factors used above are determined by:

$$
\begin{gathered}
W_{0}^{(m)}=\frac{\lambda}{n+\lambda}, W_{0}^{(c)}=\frac{\lambda}{n+\lambda}+1-\alpha^{2}+\beta \\
W_{i}^{(m)}=W_{i}^{(c)}=\frac{1}{2(n+\lambda)}
\end{gathered}
$$

where $\beta$ is a tuning parameter used to introduce prior knowledge of the distribution of the state (generally set to 2 for Gaussian distribution). Jacobian matrices are not needed in the UKF as in the EKF one, making the UKF implementation easier. 